\title{
Relationship between serum creatinine and obesity in children in Xinjiang, China
}

\author{
X.-M. Li, Y.-T. Ma, X. Xie, Y.-N. Yang, X.-M. Li and Y.-Y. Zheng \\ Department of Coronary Artery Disease, Heart Center, \\ First Affiliated Hospital of Xinjiang Medical University, Urumqi, China \\ Corresponding author: Y.-T. MA \\ E-mail: myt_xj@163.com / myt_xj@sina.com
}

Genet. Mol. Res. 13 (2): 2409-2416 (2014)

Received December 4, 2012

Accepted June 27, 2013

Published April 3, 2014

DOI http://dx.doi.org/10.4238/2014.April.3.13

\begin{abstract}
This study was designed to analyze the relationship between serum creatinine and body mass index in children in Xinjiang, China. We used a stratified sampling method to select 5222 children aged 6-17 years in 3 areas in Xinjiang and then measured serum creatinine with an enzymatic method. Our analysis showed that the mean serum creatinine of the various age groups differed, and the reference value increased gradually with age. In the groups with subjects older than 10 years, the serum creatinine values had a positive correlation with body mass index $(r=0.016,10$ - and 11-year-olds; $r=0.177,12$ - and 13-yearolds; $r=0.314,14$ - and 15 -year-olds; $r=0.380,16$ - and 17-year-olds; $\mathrm{P}<0.05)$. In the multivariate regression analysis model, the positive relationship existed even after we removed influencing factors such as blood sugar and cholesterol ( $\beta=0.041,10$ - and 11-year-olds; $\beta=0.081$, 12 - and 13 -year-olds; $\beta=0.183,14$ - and 15 -year-olds; $\beta=0.171,16$ - and 17 -year-olds; $\mathrm{P}<0.05$ ). Obesity is an independent risk factor associated with increasing serum creatinine levels in children aged more than 10 years. Weight control is important in the protection of renal function.
\end{abstract}

Key words: Serum creatinine; Obesity; Body mass index; Children; Renal function 


\section{INTRODUCTION}

The incidence of obesity is increasing, and the number of obese children has grown rapidly with continuous improvements in living standards, reduced physical activity, and excessive dietary energy. The China Child Overweight and Obesity Collaborative Research Group revealed that in 2008, the detectable rates for overweight and obese children were 19.8 and $7.2 \%$, respectively, which are close to the values in developed countries (Ding, 2008). As the prevalence of obesity increases, so does the prevalence of its associated comorbidities. In recent years, obesity has been recognized as an important factor in kidney damage. Moreover, the obesity epidemic is associated with a parallel increase in the incidence of kidney disease (Hall et al., 2003; Fox et al., 2004; Gelber at al., 2005; Kramer et al., 2005; Hsu et al., 2006). Presently, body mass index (BMI) is one of the most generally used indicators for overweight and obesity.

Previous studies have shown that urinary albumin and serum creatinine values are highly correlated with BMI in patients with type 2 diabetes, and these values increased significantly with the increase in BMI and waist circumference (Rossi et al., 2010). Other reaction indicators of renal function like glomerular filtration rate and serum uric acid (UA) have been shown to be associated with obesity or metabolic syndrome (Ford et al., 2007; Li et al., 2010). Li et al. (2010) have found no correlation between creatinine and body mass index in their research on kidney function and obesity in adults with normal blood pressure. Serum creatinine is an important indicator that is widely used in clinical work to reflect kidney function. However, only a small number of research reports on the correlation between serum creatinine levels and obesity in children have been published. In the present study, we aimed to investigate the correlation between serum creatinine levels and obesity in children in Xinjiang, China.

\section{MATERIAL AND METHODS}

\section{Subjects}

This epidemiological study of children was a multiethnic, community-based, and cross-sectional study designed to investigate the prevalence, incidence, and risk factors for cardiovascular, metabolic, and kidney diseases in children in the Han, Uygur, and Kazak ethnicities living in northwest China. We used a stratified sampling method to select a representative sample of the children of Chinese Hans, Uygurs, and Kazakhs in 3 cities (Hetian, Aletai, and Kashi). A total of 5222 individuals were analyzed in the study. This study was conducted in accordance with the Declaration of Helsinki guidelines. Written informed consent was obtained from the parents of each subject according to a protocol approved by the ethics committee of the First Affiliated Hospital of the Xinjiang Medical University.

\section{Data collection}

The demographic and serological data were reviewed simultaneously. The children surveyed were required to fast for $12 \mathrm{~h}$. Information on demographic characteris- 
tics included age, gender, education, address, and ethnicity. The measurements included height, weight, waist circumference, abdominal circumference, hip circumference, systolic blood pressure, and diastolic blood pressure. All participants underwent a standardized physical examination performed by experienced research staff. Height, waist circumference, abdominal circumference, and hip circumference were measured to the nearest 0.1 $\mathrm{cm}$, and weight was measured with a standard scale in the upright position to the nearest $0.1 \mathrm{~kg}$. BMI was calculated as weight $(\mathrm{kg})$ divided by height squared $\left(\mathrm{m}^{2}\right)$. The diagnoses of overweight and obesity in children were made according to the cutoff BMI announced in 2009 by the China Obesity Task Force as the screening criteria for children aged 0-18 years (Li et al., 2009).

Serum was separated from the samples within $30 \mathrm{~min}$ and stored at $-80^{\circ} \mathrm{C}$ until analysis. We measured the serum concentrations of UA, creatinine (enzymatic method), fasting glucose (GLU), triglycerides (TG), cholesterol (CHOL), high-density lipoprotein CHOL (HDL-C), and low-density lipoprotein CHOL (LDL-C) using equipment for chemical analysis (Dimension AR/AVL Clinical Chemistry System, Newark, NJ, USA) at the clinical laboratory of the First Affiliated Hospital of the Xinjiang Medical University.

\section{Statistical analysis}

Data analysis was performed using SPSS for Windows version 17.0 (SPSS, Inc., Chicago, IL, USA). The subjects were divided into the following groups based on age: 6-7 years, $\mathrm{N}=167 ; 8-9$ years, $\mathrm{N}=630 ; 10-11$ years, $\mathrm{N}=1385 ; 12-13$ years, $\mathrm{N}=2032$; 14 15 years, $N=897 ; 16-17$ years, $N=111$. Demographic and clinical characteristics of the study population were expressed as means \pm standard deviations. Bivariate associations between the variables were examined using Pearson's correlation coefficients. All parametric data were analyzed using the Student $t$-test, analysis of variance, and analysis of covariance, where appropriate. Multivariable linear regression was used to assess whether the BMI was an independent predictor of increases in creatinine. Other influencing factors include blood UA, GLU, TG, CHOL, HDL-C, and LDL-C. Statistical significance was determined at $\mathrm{P}<0.05$.

\section{RESULTS}

The total number of participants was 5222 , and $47.3 \%$ were male. Our analysis showed that the mean values of serum creatinine differed in the various age groups (Table 1). The mean values increased gradually with age: $37.74 \mu \mathrm{M}$ for $6-7$ years, $38.89 \mu \mathrm{M}$ for $8-9$ years, $39.12 \mu \mathrm{M}$ for $10-11$ years, $39.40 \mu \mathrm{M}$ for $12-13$ years, $46.32 \mu \mathrm{M}$ for $14-15$ years, and $57.33 \mu \mathrm{M}$ for $16-17$ years.

Correlation analysis showed that the factors associated with serum creatinine in the age groups differed (Table 2). However, the serum creatinine levels were significantly directly correlated with BMI, UA, GLU, CHOL, HDL-C, and LDL-C in the groups of children older than 10 years. In addition, the correlation coefficient between serum creatinine and BMI gradually increased with age $(r=0.016,10-11$ years; $r=0.177,12-13$ years; $r=0.314,14-15$ years; and $\mathrm{r}=0.380,16-17$ years; $\mathrm{P}<0.05$ ). This relationship is depicted in a scatter diagram of all subjects grouped according to age (Figure 1). 


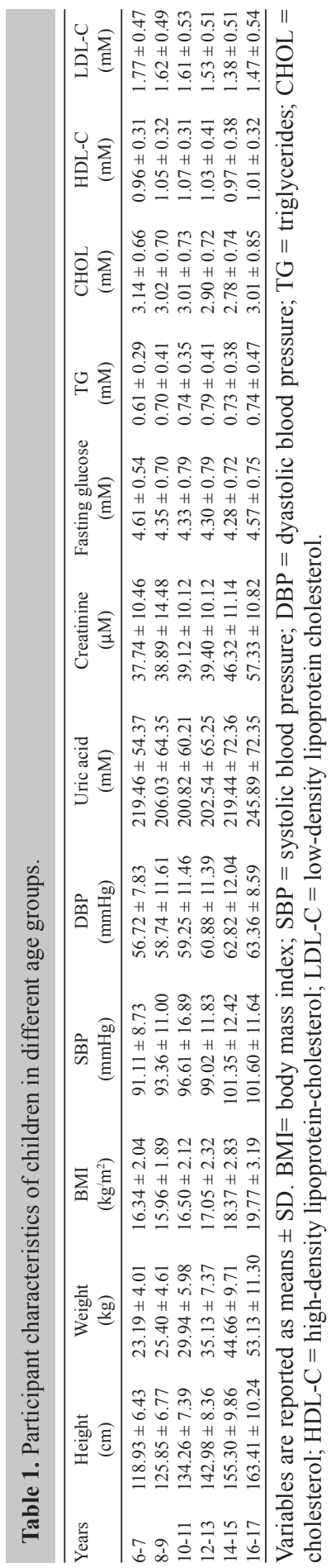


Table 2. Correlation coefficient between creatinine and SBP, BMI, uric acid, fasting glucose, TG, CHOL, HDL-C, and LDL-C.

\begin{tabular}{|c|c|c|c|c|c|c|c|c|}
\hline Years & SBP & BMI & Uric acid & Fasting glucose & TG & CHOL & HDL-C & LDL-C \\
\hline $6-7$ & 0.045 & -0.150 & $0.433^{*}$ & $0.519^{*}$ & 0.110 & $0.579^{*}$ & $0.445^{*}$ & $0.275^{*}$ \\
\hline $8-9$ & $0.095^{*}$ & 0.012 & $0.392 *$ & $0.191 *$ & $0.117^{*}$ & $0.353^{*}$ & $0.144 *$ & $0.246^{*}$ \\
\hline $10-11$ & 0.027 & $0.160^{*}$ & $0.484 *$ & $0.326^{*}$ & $0.135^{*}$ & $0.579^{*}$ & $0.353^{*}$ & $0.404 *$ \\
\hline $12-13$ & $0.075^{*}$ & $0.179 *$ & $0.552 *$ & $0.245^{*}$ & $0.091 *$ & $0.503 *$ & $0.206^{*}$ & $0.345^{*}$ \\
\hline $14-15$ & 0.009 & $0.314^{*}$ & $0.569 *$ & $0.487 *$ & -0.039 & $0.405^{*}$ & $0.096^{*}$ & $0.310^{*}$ \\
\hline $16-17$ & 0.165 & $0.380^{*}$ & $0.583^{*}$ & $0.686^{*}$ & $0.235 *$ & $0.507 *$ & $0.295 *$ & $0.408^{*}$ \\
\hline
\end{tabular}

$\mathrm{SBP}=$ systolic blood pressure $\mathrm{BMI}=$ body mass index; $\mathrm{TG}=$ triglycerides; $\mathrm{CHOL}=$ cholesterol; $\mathrm{HDL}-\mathrm{C}=$ highdensity lipoprotein $\mathrm{CHOL}$; $\mathrm{LDL}=$ low-density lipoprotein $\mathrm{CHOL} . \mathrm{P}<0.05$.
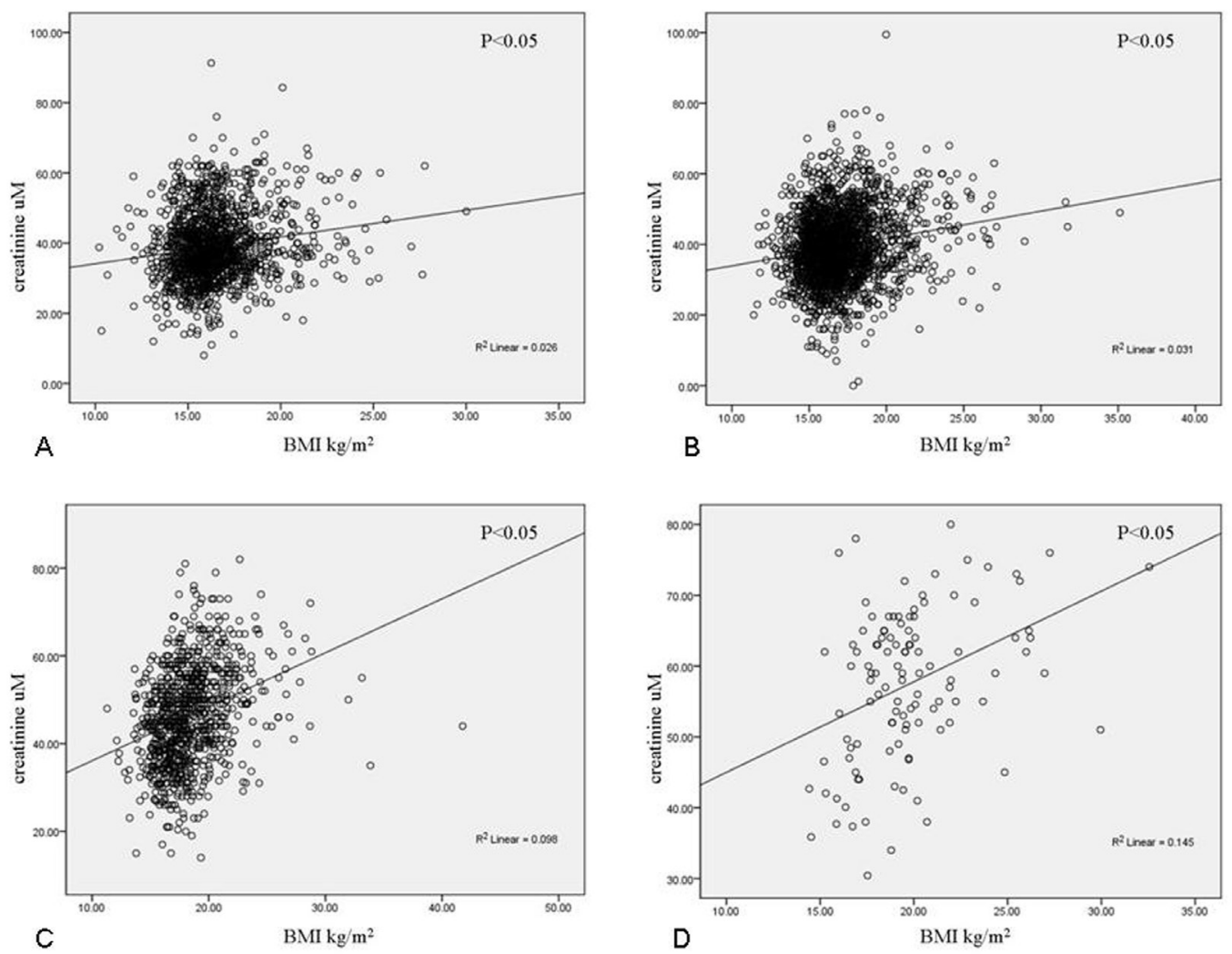

Figure 1. Relation of creatinine to body mass index (BMI). A. Scatter plot showing the correlation between creatinine and BMI in children aged 10-11 years. B. Scatter plot showing the correlation between creatinine and BMI in children aged 12-13 years. C. Scatter plot showing the correlation between creatinine and BMI in children aged 14-15 years. D. Scatter plot showing the correlation between creatinine and BMI in children aged 16-17 years.

We used multivariable linear regression to assess whether the BMI was an independent predictor of the increase in creatinine. The result revealed that a positive relationship between these parameters existed even after the removal of influencing factors such as UA, GLU, TG, CHOL, HDL-C, and LDL-C in children older than 10 years $(\beta=0.041,10-11$ years; $\beta=0.081,12-13$ years; $\beta=0.183,14-15$ years; $\beta=0.171,16-17$ years; $P<0.05$; Table 3 ). 


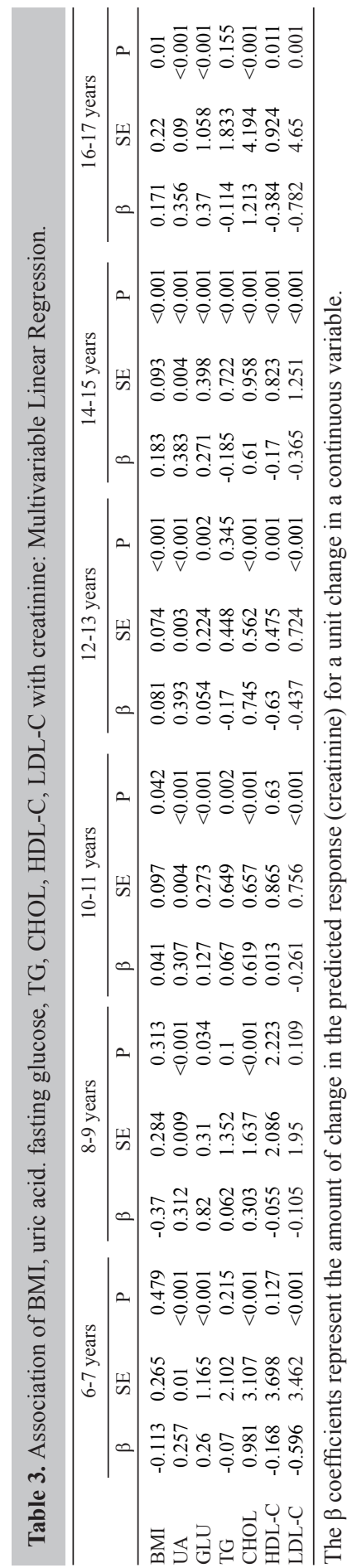




\section{DISCUSSION}

Creatinine is a small molecule can be filtered through the renal glomeruli; only a minute amount of absorption occurs in the renal tubular region. Until recently, this value was still widely used in clinical work to assess kidney function. Our study showed that creatinine value increased gradually with age, a finding matched by those of Uemura et al. (2011), who reported that serum creatinine concentrations in Japanese children differ significantly according to gender and age.

Our analysis showed that serum creatinine level was significantly directly correlated with the BMI in children older than 10 years. This positive relationship existed even after removing the influence of the covariate parameters. Currently, BMI is the accepted standard measure of overweight and obesity for adults and children aged 2 years and older. Previous studies have reported an association between the mechanism of renal dysfunction and obesity. Obesity is linked to several functional and structural alterations in the kidney. Renal diseases mainly proceed from hemodynamic abnormalities, obesity-related dysfunction, metabolic abnormalities, and mast cellrelated kidney inflammation mechanisms (Rao and Zhang et al., 2006).

Excessive body weight can lead to diabetes mellitus and hypertension. These 2 major consequences of obesity account for $70 \%$ of end-stage renal disease (Hall, 2003; Hall et al., 2003). A study in mice by do Carmo et al. (2009) revealed that kidney dysfunction owing to obesity may have a significant positive correlation with increases in blood pressure. A study by Pinto-Sietsma et al. (2003) revealed that the relationship between BMI and end-stage renal disease persists even when adjusted for diabetes and hypertension. Ishizaka et al. (2009) found that BMI is one of the predictors of chronic kidney disease, and this finding is supported by other studies. Obesity contributes to the deterioration of renal function in patients with underlying renal disease (Praga et al., 2000, 2001). The animal experiments of Henegar at al. (2001) showed that obesity-induced glomerular damage can be particularly rapid. The organizational, biochemical, and renal functions of dogs fed high-fat meals changed after just 7-9 weeks (Henegar et al., 2001). Furthermore, many researchers have demonstrated that reductions in body weight can reverse renal damage (Adelman et al., 2001; Masuo et al., 2011; Sheen and Shen, 2011). Obesity is clearly an independent risk factor for kidney damage.

Our study showed that serum creatinine level was significantly directly correlated with BMI. BMI was an independent predictor of creatinine increase in children aged more than 10 years. The prevention of childhood obesity is exceptionally important in the protection of renal function.

\section{Conflicts of interest}

The authors declare no conflict of interest.

\section{ACKNOWLEDGMENTS}

Research supported by the Xinjiang Autonomous Region Science and Technology Projects (\#201233138).

\section{REFERENCES}

Adelman RD, Restaino IG, Alon US and Blowey DL (2001). Proteinuria and focal segmental glomerulosclerosis in severely obese adolescents. J. Pediatr. 138: 481-485. 
Ding ZY (2008). National epidemiological survey on childhood obesity (2006). Zhonghua Er Ke Za Zhi. 46: 179-184. do Carmo JM, Tallam LS, Roberts JV, Brandon EL, et al. (2009). Impact of obesity on renal structure and function in the presence and absence of hypertension: evidence from melanocortin-4 receptor-deficient mice. Am. J. Physiol. Regul. Integr. Comp Physiol. 297: R803-R812.

Ford ES, Li C, Cook S and Choi HK (2007). Serum concentrations of uric acid and the metabolic syndrome among US children and adolescents. Circulation 115: 2526-2532.

Fox CS, Larson MG, Leip EP, Culleton B, et al. (2004). Predictors of new-onset kidney disease in a community-based population. JAMA 291: 844-850.

Gelber RP, Kurth T, Kausz AT, Manson JE, et al. (2005). Association between body mass index and CKD in apparently healthy men. Am. J. Kidney Dis. 46: 871-880.

Hall JE (2003). The kidney, hypertension, and obesity. Hypertension 41: 625-633.

Hall JE, Kuo JJ, da Silva AA, de Paula RB, et al. (2003). Obesity-associated hypertension and kidney disease. Curr. Opin. Nephrol. Hypertens. 12: 195-200.

Henegar JR, Bigler SA, Henegar LK, Tyagi SC, et al. (2001). Functional and structural changes in the kidney in the early stages of obesity. J. Am. Soc. Nephrol. 12: 1211-1217.

Hsu CY, McCulloch CE, Iribarren C, Darbinian J, et al. (2006). Body mass index and risk for end-stage renal disease. Ann. Intern. Med. 144: 21-28.

Ishizaka Y, Ishizaka N, Tani M, Toda A, et al. (2009). Association between chances in obesity parameters and incidence of chronic kidney disease in Japanese individuals. Kidney Blood Press Res. 32: 141-149.

Kramer H, Luke A, Bidani A, Cao G, et al. (2005). Obesity and prevalent and incident CKD: the Hypertension Detection and Follow-Up Program. Am. J. Kidney Dis. 46: 587-594.

Li GH, Leng S, Zhao J and Zhu Y (2010). Change of renal function in overweight and obesity people with normal blood pressure. Med. Philos. Clin. Decis. Making Forum Edition 7: 457-459.

Li H, Ji CY, Zong XN and Zhang YQ (2009). Body mass index growth curves for Chinese children and adolescents aged 0 to 18 years. Zhonghua Er Ke Za Zhi. 47: 493-498.

Masuo K, Rakugi H, Ogihara T, Esler MD, et al. (2011). Effects of weight loss on renal function in overweight Japanese men. Hypertens. Res. 34: 915-921.

Pinto-Sietsma SJ, Navis G, Janssen WM, de ZD, et al. (2003). A central body fat distribution is related to renal function impairment, even in lean subjects. Am. J. Kidney Dis. 41: 733-741.

Praga M, Hernandez E, Herrero JC, Morales E, et al. (2000). Influence of obesity on the appearance of proteinuria and renal insufficiency after unilateral nephrectomy. Kidney Int. 58: 2111-2118.

Praga M, Hernandez E, Morales E, Campos AP, et al. (2001). Clinical features and long-term outcome of obesityassociated focal segmental glomerulosclerosis. Nephrol. Dial. Transplant 16: 1790-1798.

Rao XR and Zhang GH (2006). Obesity and kidney damage. Chin. J. Integr. Tradit. West. Nephrol. 10: 616-618.

Rossi MC, Nicolucci A, Pellegrini F, Comaschi M, et al. (2010). Obesity and changes in urine albumin/creatinine ratio in patients with type 2 diabetes: the DEMAND study. Nutr. Metab. Cardiovasc. Dis. 20: 110-116.

Sheen YJ and Shen WH (2011). Metabolic syndrome and renal injury. Cardiol. Res. Pract. 13: 567-589.

Uemura O, Honda M, Matsuyama T, Ishikura K, et al. (2011). Age, gender, and body length effects on reference serum creatinine levels determined by an enzymatic method in Japanese children: a multicenter study. Clin. Exp. Nephrol. 15: 694-699. 